\title{
Dos décadas de Acta Colombiana de Psicología: un análisis bibliométrico
}

\author{
Gonzalo Salas*, Ernesto L. Ravelo-Contreras ${ }^{2}$, Susana Mejía ${ }^{2}$, Romina Andrades ${ }^{3}$, Evelyn Acuña ${ }^{3}$, Felipe Espinoza ${ }^{3}$, Manuel \\ Núñez ${ }^{3}$, Miguel Barboza-Palomino ${ }^{4}$, José Ventura-León ${ }^{4}$, Tomás Caycho-Rodríguez ${ }^{4}$, Andrés M. Pérez-Acosta ${ }^{5}$. \\ ${ }^{1}$ Universidad Católica del Maule, Chile, ${ }^{2}$ Universidad Católica de Colombia, ${ }^{3}$ Universidad Católica del Maule, Chile, ${ }^{4}$ Universidad Privada \\ del Norte, Perú, ${ }^{5}$ Universidad del Rosario, Colombia.
}

Recibido, marzo 15/2018

Concepto de evaluación, abril 4/2018

Aceptado, abril 23/2018

\begin{abstract}
Referencia: Salas, G., Ravelo-Contreras, E.L., Mejía, S., Andrades, R., Acuña, E., Espinoza, F., Núñez, M., Barboza-Palomino, M., Ventura-León, J., Caycho-Rodríguez, T., \& Pérez-Acosta, A.M. (2018). Dos décadas de Acta Colombiana de Psicología: Un análisis bibliométrico. Acta colombiana de Psicología, 21(2), 13-25. doi: http://www.dx.doi.org/10.14718/ ACP.2018.21.2.2
\end{abstract}

Resumen

\section{Two decades of Acta Colombiana de Psicología: a bibliometric analysis ${ }^{*}$}

\begin{abstract}
The aim of the present study was to conduct a bibliometric analysis of the journal Acta Colombiana de Psicología, by virtue of its two decades of uninterrupted work (1998-2017). Initially, historical elements are described in the trajectory of the journal and its chronological evolution is revealed from a database that in its final sample took into account 388 scientific articles. Subsequently, the appraisal of the productivity indices (Lotka) as well as of the topics and methodologies used was carried out. Likewise, an appraisal of the collaboration indicators and the relationships between the authors with respect to the signatory countries was performed. The results highlight the large number of papers from Mexican institutions, a sustained increase in collaboration is visualized -which is evident in the Lawani and Subramanyam indices-, and it is found that the largest amount of papers focused on the areas of clinical and health psychology. The analysis undertaken allows showing what the journal has achieved throughout two decades of scientific work during which it contributed fundamentally to the development of psychology in Latin America.

Key words: bibliometrics, indicators, scientific production, journal, psychology.
\end{abstract}

\footnotetext{
* Departamento de Psicología. Facultad de Ciencias de la Salud. Universidad Católica del Maule, Talca, Chile. +56712203398 gsalas@ucm.cl

Los autores dedicamos este artículo a la memoria de Carlos Vargas Ordóñez (1938-2018), Decano de la Facultad de Psicología de la Universidad Católica de Colombia, Director de la revista Acta Colombiana de Psicología, desde su fundación (1998). Su particular equilibrio entre gran calidad humana y enorme capacidad de gestión también son responsables del éxito alcanzado por esta revista científica.
} 


\title{
Duas décadas de Acta Colombiana de Psicología: uma análise bibliométrica
}

\begin{abstract}
Resumo
O objetivo do presente estudo foi realizar uma análise bibliométrica da revista Acta Colombiana de Psicología, devido a suas duas décadas de trabalho ininterrupto (1998-2017). Inicialmente, descrevem-se elementos históricos na trajetória da revista e revela-se sua evolução cronológica a partir de uma base de dados que considerou 388 artigos científicos em sua amostra final. Posteriormente, foram realizadas as análises dos índices de produtividade (Lotka) e das temáticas e metodologias utilizadas. Além disso, foi desenvolvida uma análise dos indicadores de colaboração e das relações entre os autores com respeito aos países signatários. Nos resultados, destaca-se a grande quantidade de trabalhos provenientes de instituições mexicanas, visualiza-se uma alta prolongada na colaboração — o que se evidencia nos índices de Lawani e Subramanyam -, e nota-se que a maior quantidade de trabalhos concentrou-se nas áreas de psicologia clínica e da saúde. A análise realizada permite exibir o feito da revista ao longo de duas décadas de trabalho científico nas quais colaborou de maneira fundamental para o desenvolvimento da psicologia na América espanhola.

Palavras-chave: bibliometria, indicadores, produção científica, psicologia, revista.
\end{abstract}

\section{INTRODUCCIÓN}

La psicología científica en Colombia nace conectada a los campos de la educación, la medicina y el hipnotismo, siendo sobre este último la publicación del primer artículo científico, a cargo del Dr. Proto Gómez, en el año 1888 (Ardila, 1967). Desde finales del siglo XIX hasta inicios del siglo XX la psicología se estableció en el país como un campo de estudio para los investigadores colombianos de la época, con temas que respondían a las influencias intelectuales de los movimientos postcoloniales de aquel entonces (Oviedo, 2012). Sin embargo, recién a mediados del siglo XX la psicología comenzó su formación profesional cuando en 1947 la española Mercedes Rodrigo fundó el Instituto de Psicología Aplicada en la Universidad Nacional de Colombia (Ardila, 1967; Giraldo \& Rodriguez, 1997), al mismo tiempo que en Chile se empezaba con la formación de psicólogos mediante el Curso Especial de Psicología de la Universidad de Chile (Salas, 2014).

Si bien la historia de las revistas científicas de psicología a nivel mundial tiene su origen a finales del siglo XIX (Polanco-Carrasco, Gallegos, Salas \& López-López, 2017), en Latinoamérica ocurre en diversos momentos del siglo $\mathrm{XX}, \mathrm{y}$, para el caso colombiano, la primera revista científica en crearse fue la Revista de Psicología, en 1956 -gracias al trabajo del Dr. Luis Jaime Sánchez-y, posteriormente, la Revista Latinoamericana de Psicología, en 1969 -por el Dr. Rubén Ardila-(Dominguez Blanco \& Duque, 1993; Giraldo \& Rodriguez, 1997), revista crucial para el desarrollo de la psicología latinoamericana (López-López \& Calvache, 1998), que en sus inicios fue considerada como una idea ambiciosa y en cuya prosperidad pocos creían (Gallegos, 2010).
Las revistas científicas son un compendio del conocimiento que se elabora en una región específica y cuya producción favorece la apertura del conocimiento generado al interior de determinados espacios científicos, potencia el desarrollo de los países y entrega luces sobre el contexto en el que se desarrollan (Pire, 2015; Vera-Villarroel, López-López, Lillo \& Silva, 2011). Sin embargo, debido a la gran cantidad de artículos que las revistas almacenan en sus archivos, resulta necesario crear y utilizar herramientas que permitan cuantificar la producción de ciencia mediante, por ejemplo, estrategias como los análisis por medio de bibliometrías (Buela-Casal \& López, 2005; Zych \& Buela-Casal, 2010).

Este tipo de estrategias -las bibliometrías- han sido aplicadas a diversas revistas, como es el caso del Journal of Cross Cultural Psychology [JCCP], el cual ha sido relevado en diversas oportunidades (Best \& Everett, 2010; Brouwers, Van Hemert, Breugelmans, Van de, \& Vijver, 2004; Lonner, 1980); el Journal of Environmental Psychology [JEP] (Milfont \& Page, 2013); la revista chilena Psykhe (Salas et al., 2017); e, incluso, la revista objeto del presente estudio, Acta Colombiana de Psicología, en la que se realizó un estudio sobre las publicaciones del periodo comprendido entre el 2010 y el 2014 (Ravelo, Mejía, \& González, 2016); todo lo cual contribuye a la comprensión de la dinámica de las revistas en el contexto científico.

Teniendo en cuenta lo anterior, el objetivo del presente trabajo es realizar un análisis bibliométrico y, adicionalmente, otorgar una mirada generalizada del pasado, el estado actual y las proyecciones en cuanto a la historia y producción científica de la revista Acta Colombiana de Psicología [de aquí en adelante $A c t a]$ durante sus 20 años de trayectoria 
(1998-2017), con el fin de identificar y analizar diversos indicadores bibliométricos.

\section{Historia y actualidad de Acta}

Acta es editada por la Facultad de Psicología de la Universidad Católica de Colombia con la finalidad de contribuir al desarrollo de la ciencia psicológica. Sus publicaciones están dirigidas principalmente a problemas relevantes en la psicología científica, y su alcance esperado apunta a llegar a universidades, entidades privadas y el Estado, así como a centros de salud y de educación. Desde sus inicios, esta revista fue concebida como una herramienta para todas aquellas personas interesadas en estudiar y compartir experiencias académicas en psicología y en algunas temáticas médicas, como al identificar las necesidades de la sociedad moderna, los nuevos avances en dichas áreas, y estudiar sus aplicaciones en diferentes campos (Vargas, 1998); y esto se mantuvo en su esencia, a pesar de que hubo algunos cambios en las diversas etapas de desarrollo de Acta.

Específicamente, en octubre de 1998 se publicó el primer número de la revista - con una periodicidad anual-, dedicado al tema de la investigación psicológica de las adicciones con motivo de la creación del Centro de Estudios e Investigaciones sobre las Adicciones de la institución (FlórezAlarcón, 1998). Acta apareció en un momento en el que existían pocos títulos consolidados de revistas psicológicas en el país, y en donde el principal referente era la ya citada Revista Latinoamericana - que celebraba su tercera década por aquél entonces-(Ardila, 1998), la única revista científica colombiana incluida en los índices de citación del Institute for Scientific Information (ISI). En ese momento, el panorama nacional de revistas psicológicas vigentes e indexadas estaba conformado también por los siguientes títulos: Avances en Psicología Clínica Latinoamericana (fundada en 1982), Revista Interamericana de Psicología Ocupacional (en 1982), Revista Colombiana de Psicología (en 1992) y Suma Psicológica (en 1994) (PérezAcosta \& Perilla Toro, 1996).

En cuanto a la investigación psicológica, en aquella época Colombia comenzaba a generar producción de calidad, lo que justificó la creación de nuevos medios locales de publicación (Ardila \& Pérez-Acosta, 2010), además de grupos y centros de investigación que fueran reconocidos por el Departamento Administrativo de Ciencia, Tecnología e Innovación-Colciencias-. Con esto, se forjaron laboratorios psicológicos, posgrados de investigación (tanto maestrías como doctorados), eventos académicos de alcance internacional y premios de investigación (Pérez-Acosta \& Perilla Toro, 2006). En ese momento, la investigación psicológica en el país se centró en las áreas de psicología social, análisis experimental del comportamiento, neuropsicología y psicobiología, medición y evaluación, aprendizaje, cognición, psicología del desarrollo evolutivo, psicología clínica, psicología de la salud, el mundo del trabajo, y la psicología de la personalidad (Ardila, 2013).

Asimismo, aquel momento de maduración de la investigación se vivió también en la Facultad de Psicología de la Universidad Católica de Colombia (Vargas, 1998), que ya había completado 25 años con un desarrollo creciente en numerosas áreas diferenciadas, como en psicología educativa, psicología organizacional, psicología jurídica, psicología clínica, medición y evaluación psicológica, e, incluso, en psicología básica; por lo que, no casualmente, los números subsiguientes de Acta (desde el año 2000 cambió su periodicidad a semestral) estuvieron dedicados de forma monográfica a la investigación en dichas áreas. De igual forma, estos desarrollos fueron sustentando la creación de programas de posgrado en la institución tanto a nivel de especialización como de maestría y doctorado, tal como se fue informando en las sucesivas notas de la sección "información institucional" de la revista.

El Decano de la Facultad de Psicología de ese entonces, Carlos Vargas Ordóñez, confió la responsabilidad editorial de la nueva revista al Director de Investigaciones de la Facultad, el Dr. Ernesto Ravelo, quien sigue siendo el Editor de la misma en la actualidad. El Dr. Ravelo se apoyó en el Comité Editorial de la Facultad, conformado en sus primeros años, además del Decano, por Héctor Balmes Ocampo, Arturo Bríñez Horta, Leonor Delgado Abella, Luis Flórez Alarcón, Nohelia Hewitt Ramírez, Patricia Hernández Zubieta, Marta Lozano Ardila, Judit Martínez, Andrés M. Pérez-Acosta, José Antonio Sánchez González y Nancy Vargas Espinosa, profesores de la Facultad.

Entre 1998 y 2005, Acta tuvo una primera etapa, caracterizada por la publicación de números temáticos respaldados por los investigadores de la institución en cada uno de los siguientes campos:

- Investigación en adicciones y violencia: números 1 (1998), 4 (2000) y 9 (2003).

- Psicología educativa: números 2 (1999) y 3 (2000).

- Medición y evaluación en psicología: número 5 (2001).

- Psicología jurídica: número 6 (2001).

- Psicología clínica: números 7 (2002) y 12 (2005).

- Análisis del comportamiento humano: número 8 (2002).

- Psicología básica: número 10 (2003).

- Psicología organizacional: número 11 (2004).

- Psicología social: número 12 (2004).

Y durante esa primera etapa, se fueron diferenciando varias responsabilidades y comités dentro de la revista:

- Director: Carlos Vargas Ordóñez.

- Editor: Ernesto Ravelo Contreras.

- Coordinación editorial: Stella Valbuena García. 
- Comité editorial (2005): Luis Flórez Alarcón, Alba Lucía Meneses Báez, Rosalía Montealegre Hurtado, Carlos Pardo Adames y Andrés M. Pérez-Acosta.

- Consejo editorial (2005): Delcy Elena Cáceres, Leonidas Castro Camacho, Duilio Cruz Becerra, Lucía Chávez Correal, César Rey, Beatriz Molano y Luz Anyela Morales Quintero.

- Comité científico (2005): Rubén Ardila, Alfredo Ardila, Vicente E. Caballo, Maria Helena Leite Hunziker, Héctor Martínez Sánchez, Maritza Montero, Alba E. Mustaca, José I. Navarro Guzmán, Ricardo Pellón, Wanda Rodríguez Arocho y Andrés Santamaría Santigosa.

Gracias al liderazgo y a la competente y juiciosa gestión del Editor, la revista también logró en aquella época su inclusión en el Índice Bibliográfico Nacional de revistas científicas, Publindex, de Colciencias (Vargas, 2004). Reconocimiento que comenzó una carrera de visibilidad e impacto de la revista, aún creciente, gracias a su inclusión en bases de datos internacionalmente reconocidas, como lo son Scopus (de Elsevier, fluctuando entre Q4 y Q3 desde 2008 hasta la fecha), SciELO Citation Index (Clarivate Analytics), PsycINFO (American Psychological Association), Psicodoc (Colegio Oficial de Psicólogos de Madrid, España), DOAJ (Universidad de Lund, Suecia), Redalyc (Universidad Autónoma del Estado de México), Dialnet (Universidad de la Rioja, España), CLASE(Universidad Nacional Autónoma de México) y LILACS (BIREME, Brasil), entre otras.

La temprana indexación de la revista tuvo cuatro consecuencias que se apreciaron en los años 2005 y 2006 : (a) se detuvo la secuencia de números temáticos para dar paso a números regulares conformados por artículos sometidos de forma independiente (véase número 14 de 2005); (b) se comenzaron a publicar más de diez artículos por número (antes se publicaban típicamente números con siete artículos); (c) se dio paso a la publicación en lengua portuguesa (Sebastiani \& Maia, 2006), lo cual reforzó el sometimiento en dicho idioma, especialmente desde Brasil; y (d) se presentaron notables cambios en el formato (estándar internacional ISO, tamaño carta, color uniforme, entre otros detalles) y la secuencia, que desde 2006 pasó a ser de volumen anual a de dos números por volumen.

En 2008, luego de su primera década, Acta comenzó a publicar reseñas de novedades bibliográficas al estilo de otras revistas consolidadas, como la Revista Latinoamericana de Psicología, la Revista Interamericana de Psicología o Psicothema. La primera reseña fue del libro Neurociencia, violencia y sistema de justicia, de Feggy Ostrosky-Solís, elaborada por la Dra. Luz Anyela Morales Quintero, miembro del Comité Científico y actual profesora de la Benemérita Universidad Autónoma de Puebla (Morales Quintero, 2008) -desde ese momento, la Dra. Morales es responsable de la coordinación y proceso de selección de dichas reseñas-. Adicionalmente, otra novedad importante en ese mismo año fue la inclusión de los resúmenes y palabras clave en portugués, con el fin de facilitar la visibilidad y el impacto de la revista en el mundo luso-parlante.

Hacia 2012, cuando cumplió 15 años, Acta ya había superado completamente su original carácter institucional y nacional, y se convirtió en una publicación plenamente internacional. Señal inequívoca es que ninguno de sus quince artículos tenía autores de la Universidad Católica de Colombia, sino que eran propuestos desde instituciones de Portugal, España, México, Chile y Argentina, además de otras también de Colombia; lo cual contrastó de manera importante con el primer número de la revista (1998), que incluía cuatro artículos locales de la institución, un artículo de otra institución nacional y el cierre de un artículo de un autor peruano. De igual forma, el equipo de la revista se diversificó con la adición de nuevos cargos, comités y miembros extranjeros, aunque siempre guardó la continuidad que requiere cualquier revista científica exitosa:

- Director: Carlos Vargas Ordóñez.

- Editor: Ernesto Ravelo Contreras.

- Directora editorial: Stella Valbuena García.

- Comité editorial: Luis Flórez Alarcón, Eulalia María Chaves, María del Rocío Hernández Pozo, Francisco J. Labrador Encinas, Héctor Martínez Sánchez, Ricardo Pellón y Andrés M. Pérez-Acosta.

- Consejo editorial: Delcy Elena Cáceres, Leonidas Castro Camacho, Alba Lucía Meneses Báez, Beatriz Molano García, Rosalía Montealegre Hurtado, Maritza Montero, Carlos Pardo Adames y Ricardo Werner Sebastiani.

- Comité científico: Rubén Ardila, Alfredo Ardila, Eric García-López, Luz Anyela Morales Quintero, Alba E. Mustaca, José I. Navarro Guzmán, César Rey Anacona, Wanda Rodríguez Arocho, Andrés Santamaría Santigosa y Stefano Vinaccia.

En 2013, Acta dio otro importante paso en su visibilidad: comenzó a publicar los artículos con código DOI (Digital Object Identifier), con lo que se facilita la ubicación individual de los artículos en el portal electrónico de la revista. Asimismo, en 2016 al DOI se unió la política editorial de publicar los artículos en inglés, además del idioma original del artículo (Ravelo, 2017).

De esta manera, la revista se sumó a un exitoso conjunto de títulos que, gracias a su visibilidad e impacto, convirtieron a Colombia en una meca para los autores iberoamericanos en el área (Gutiérrez, Pérez-Acosta \& Plata-Caviedes, 2009), sobre todo porque, en los últimos 10 años, la pionera Revista Latinoamericana de Psicología quedó definitivamente acompañada de otros influyentes títulos nacionales, como Universitas Psychologica, Avances en Psicología 
Latinoamericana, Suma Psicológica, International Journal of Psychological Research, Diversitas: Perspectivas en Psicología, Revista Colombiana de Psicología, Psicología desde el Caribe, Revista CES Psicología, y Psychologia: Avances de la Disciplina, entre otras (Ardila \& PérezAcosta, 2010).

\section{Análisis bibliométrico}

El análisis bibliométrico es el estudio cuantitativo del material bibliográfico con el que es posible hacer una clasificación de artículos, autores y revistas a fin de proporcionar un panorama general de un determinado campo de investigación (Merigó, Pedrycz, Weber \& de la Sotta, 2018) y contribuir a la comunidad científica al informar sobre los aportes complementarios en las diversas áreas en las que se aplica este tipo de análisis (Gorbea Portal, 2016). En este sentido, la investigación en el campo de las bibliometrías ha sido un importante vehículo para el análisis de la información científica en una amplia diversidad de campos disciplinares, tal como ha sucedido en el ámbito de la literatura (Tsay \& Li, 2017), la medicina (Sweileh, 2017; Sweileh, Al-Jabi, Sawalha, AbuTaha \& Zyoud, 2016) y el trabajo social (Ho, 2014), entre otras disciplinas de estudio.

La elaboración de una bibliometría permite utilizar y aplicar elementos matemáticos-estadísticos a un campo específico de la ciencia, lo que facilita la organización de la bibliometría en distintas categorías, como lo son la productividad de las revistas, su impacto en la comunidad científica, las colaboraciones entre científicos o instituciones y su distribución entre distintas bases de datos (Bordons \& Zulueta, 1999; Pritchard, 1969; Spinak, 1998). De esta forma, la bibliometría es una herramienta crucial a la hora de elaborar una sistematización práctica de la producción de conocimiento.

Específicamente, entre los indicadores de actividad de este tipo de análisis se encuentran el número y distribución de publicaciones, la productividad, la dispersión de las publicaciones, la colaboración en las publicaciones, la vida media de la citación -o envejecimiento-, y las conexiones entre autores; indicadores que facilitan la visualización de la situación actual de la ciencia, y que, en el caso de los indicadores de impacto, sirven para estimar la relevancia de los autores, trabajos o revistas (Camps, 2008).

\section{MÉTODO}

\section{Tipo de estudio}

En este trabajo, se desarrolló un estudio bibliométrico de carácter descriptivo a partir del análisis de los artículos publicados por una revista científica (Montero \& León, 2007). Específicamente, con este análisis se relevan una serie de indicadores, entre los cuales se encuentran, por ejemplo, el número total de artículos, el sexo de los autores, el idioma de la publicación, los indicadores de productividad (Lotka), los índices de colaboración (Subramanyam y Lawani), el país de afiliación, las áreas temáticas y las metodologías implementadas.

\section{Universo de estudio}

Con un total de 455 documentos publicados en Acta, se aplicó un filtro a partir del cual se excluyeron todos los trabajos que no fueron clasificados propiamente como artículos científicos. De acuerdo con este criterio de exclusión, se segregaron los documentos etiquetados como editoriales, información institucional, ponencias, presentaciones y reseñas, por lo que finalmente se hizo el análisis con un total de 388 artículos registrados entre los años 1998 y 2017.

\section{Instrumentos}

Para el establecimiento de la base de datos a analizar, se construyó una planilla en Excel que fue alimentada con todos los documentos de la revista, en donde se aplicó el filtro descrito más arriba. Esta base de datos fue creada con la información del sitio web oficial de la revista (http:// editorial.ucatolica.edu.co/ojsucatolica/revistas_ucatolica/ index.php/acta-colombiana-psicología/).

\section{Procedimiento}

Inicialmente, se identificaron las variables a analizar, y a partir de la información entregada por cada artículo científico, se levantaron los datos con la finalidad de construir la base de datos en Excel con la que se llevó a cabo el proceso del análisis de la información.

\section{Análisis de datos}

Una vez completada la base de datos, se llevó a cabo el procesamiento y análisis de datos mediante la misma planilla Excel, complementada con el paquete IBM SPSS Statistics, versión 23. Específicamente, se realizó el análisis de la Ley de Lotka (Urbizagástegui, 2005) con el programa Excel, y se realizaron cálculos de frecuencias y porcentajes por medio del SPSS (Ravelo et al., 2016), para luego proceder a realizar las tablas y figuras correspondientes. Es importante destacar que algunos análisis se realizaron en torno a quinquenios (1998-2002, 2003-2007, 2008-2012 y 2013-2017), mientras que otros, como la colaboración entre países, fueron analizados por décadas (1998-2007 y 2008-2017). 


\section{RESULTADOS}

\section{Productividad}

En general, la revista publicó un total de 388 artículos científicos, en donde se encontró un porcentaje mayor de mujeres en la primera autoría (52 \%) -sin embargo, al considerar la totalidad de los autores, esta relación se invierte, siendo los hombres quienes presentan dicho porcentaje-; asimismo, de la totalidad de los artículos, más de dos tercios se encuentran indexados en Scopus (véase Tabla 1), lo cual indica un factor importante de visibilidad de la revista en el ámbito mundial. De igual forma, cabe resaltar que, si bien los datos muestran un número menor de artículos escritos en inglés, la revista ha realizado el esfuerzo de traducir a dicho idioma todos los artículos que fueron enviados originalmente en español o portugués, lo que demuestra un cambio de fondo en la política editorial que generará mejoras importantes en el impacto y la citación de los artículos (Di Bitteti \& Ferreras, 2017), sumado a la percepción de los investigadores de que su investigación será reconocida y recompensada (López-Navarro, Moreno, Quintanilla \& Rey-Rocha, 2015).

Tabla 1

Estadísticos descriptivos de los artículos publicados en Acta (1998-2017)

\begin{tabular}{cccc}
\hline Categoría de análisis & Total & Hombres & Mujeres \\
\hline Artículos & & & \\
$\mathrm{N}$ & 388 & $186(48 \%)$ & $202(52 \%)$ \\
Autores & 718 & $377(52 \%)$ & $346(48 \%)$ \\
Indexación & & & \\
Scopus & $264(68 \%)$ & & \\
No Scopus & $124(32 \%)$ & & \\
Idioma & & & \\
Español & $345(89 \%)$ & & \\
Inglés & $22(6 \%)$ & & \\
Portugués & $21(5 \%)$ & & \\
\hline
\end{tabular}

Por otra parte, con respecto al contraste de la Ley de Lotka sobre la productividad científica de los autores que publicaron en Acta, se utilizó en el análisis el modelo de los mínimos cuadrados (Urbizagástegui, 2005); y con el fin de contrastar la producción empírica u observada con la producción teórica o esperada se consideró solamente a los autores principales (primer autor) y se estimó la cantidad de contribuciones, lo que fue sustentado en una primera propuesta teórica (Lotka, 1926).

En la Tabla 2, la frecuencia observada indica el número de autores principales, mientras que las contribuciones por autor señalan la cantidad de publicaciones, con lo que se puede observar que 253 autores principales publicaron un solo artículo científico, mientras que 37 autores principales publicaron dos trabajos, etc. Asimismo, el valor máximo observado con respecto a la publicación de artículos por autor se dio en el caso en que un autor contribuyó con ocho artículos publicados por la revista. Tal como se señaló, el cálculo de la frecuencia esperada o teórica se realizó de acuerdo con el modelo de los mínimos cuadrados, y, en la Tabla 2, la frecuencia esperada indica un número de autores principales, lo cual se interpreta de la siguiente forma: se espera que 247 autores hayan publicado un trabajo en Acta, que 35 autores hayan publicado dos trabajos, y que 11 hayan publicado tres, y así, sucesivamente, hasta el último valor, el cual indica que se espera que un autor haya publicado ocho trabajos -valor también sugerido por la Ley de Lotka-.

El siguiente paso fue establecer si existen diferencias entre la frecuencia esperada y la frecuencia observada, y, considerando una significancia de .01, se obtuvo un valor crítico (.0828) y se estimó la desviación máxima (Dmax). A modo de interpretación, si el valor crítico es mayor a la Dmax, se acepta que la frecuencia observada se ajusta a la frecuencia esperada; mientras que, si el valor crítico es menor a la Dmax, se rechaza que la frecuencia observada se ajusta a la frecuencia esperada. Como resultado, se observa (véase Tabla 2) que el valor crítico es mayor a todos los valores de la Dmax, por lo que se afirma que existe correspondencia entre la producción científica observada y la producción científica esperada en Acta, y que, de esta forma, se cumple la Ley de Lotka (véase Figura 1). Con respecto a estos datos, es importante aclarar que, por políticas de la revista, en un mismo año solo es posible la publicación de un único artículo por autor.

Tabla 2

Diferencias entre las frecuencias observadas y las frecuencias teóricas

\begin{tabular}{cccc}
\hline $\begin{array}{c}\text { Contribuciones } \\
\text { por autor }\end{array}$ & $\begin{array}{c}\text { Frecuencia } \\
\text { observada }\end{array}$ & $\begin{array}{c}\text { Frecuencia } \\
\text { esperada }\end{array}$ & Dmax \\
\hline 1 & 253 & 247 & $.0184^{*}$ \\
2 & 37 & 35 & $.0265^{*}$ \\
3 & 9 & 11 & $.0202^{*}$ \\
4 & 4 & 5 & $.0175^{*}$ \\
5 & 2 & 3 & $.0157^{*}$ \\
6 & 0 & 2 & $.0107^{*}$ \\
7 & 0 & 1 & $.0075^{*}$ \\
8 & 1 & 1 & $.0085^{*}$ \\
Total & 306 & 303 & \\
\hline
\end{tabular}

Nota $:$ Dmax $=$ Diferencia máxima. ${ }^{*}$ Estadísticamente significativo. Valor crítico $=.0828$. 


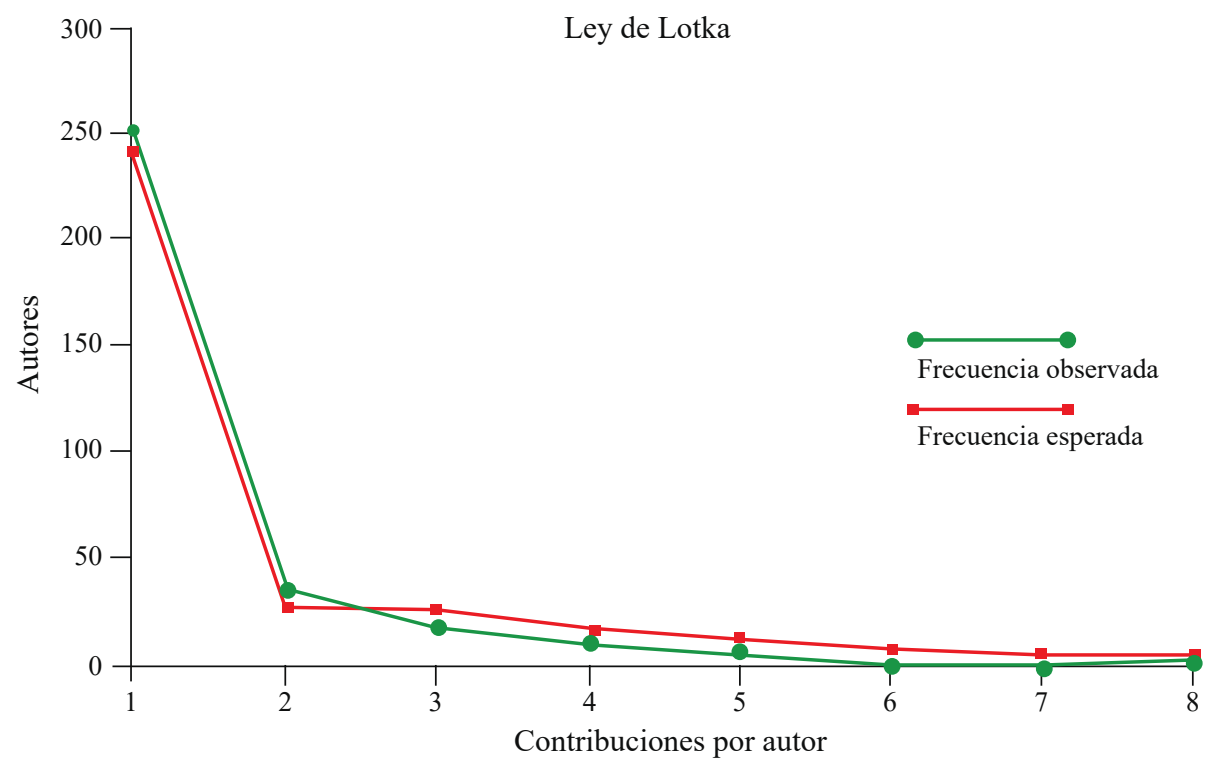

Figura 1. Ley de Lotka en las publicaciones de Acta (1998-2017).

\section{Colaboración}

En la Tabla 3 se muestra el número de autores, distribuidos según el número de autores por quinquenio. La primera evidencia detectada es que los trabajos con un solo autor han disminuido desde el primer al último quinquenio, pasando de un $60 \%$ de trabajos realizados por un autor a tan solo $12 \%$ en el último periodo, lo cual sugiere que la colaboración se ha convertido en un aspecto indispensable en la época actual.

Ahora bien, con respecto a los índices de Lawani y Subramanyam -que miden la colaboración- es posible mencionar que ambos han ido incrementándose en una curva de constante aumento. Específicamente, el índice de Lawani (1981) proporciona el peso promedio del número de autores por artículo. El valor para la revista fue de 2.58, aunque en el último periodo fue de $3.1 \mathrm{y}$ todo indica que dicho valor seguirá aumentando. Por su parte, el índice de
Subramanyam (1983) -que estudia el grado de colaboraciónrefiere que los valores más cercanos a 1 denotan una fuerte proporción de autoría múltiple, y, en este caso, muestra que la proporción de artículos con autoría múltiple para la revista es de .76, aunque, de igual forma, los resultados por quinquenio son de .88 para el último periodo.

Por otra parte, también se analizó la colaboración entre países, aunque esta variable fue estudiada en el transcurso de una década a otra. En la Figura 3a se muestran las conexiones para la década de 1998-2007 y, en la Figura 3b, las de la década de 2008-2017. Al hacer un mapeo de las figuras $3 \mathrm{a}$ y $3 \mathrm{~b}$, es posible observar con claridad el gran número de diferencias entre estos dos periodos. En la primera década se observan nexos incipientes, donde los primeros trabajos de colaboración se dan entre Colombia y España, entre Colombia y Alemania, y entre España y Chile, para finalmente existir un trabajo de colaboración

Tabla 3

Número de artículos distribuidos según número de autores por quinquenio

\begin{tabular}{cccccc}
\hline & \multicolumn{5}{c}{ Periodo } \\
\cline { 2 - 5 } & $1998-2002$ & $2003-2007$ & $2008-2012$ & $2013-2017$ & \\
\hline Número de autores & & & & \\
1 & $31(60 \%)$ & $25(29 \%)$ & $21(17 \%)$ & $16(12 \%)$ & 93 \\
2 & $12(23 \%)$ & $36(42 \%)$ & $46(38 \%)$ & $34(27 \%)$ & 128 \\
3 & $7(13 \%)$ & $6(7 \%)$ & $36(29 \%)$ & $33(26 \%)$ & 82 \\
73 & $2(4 \%)$ & $19(22 \%)$ & $19(16 \%)$ & $45(35 \%)$ & 85 \\
Total & 52 & 86 & 122 & 128 & 388 \\
\hline
\end{tabular}



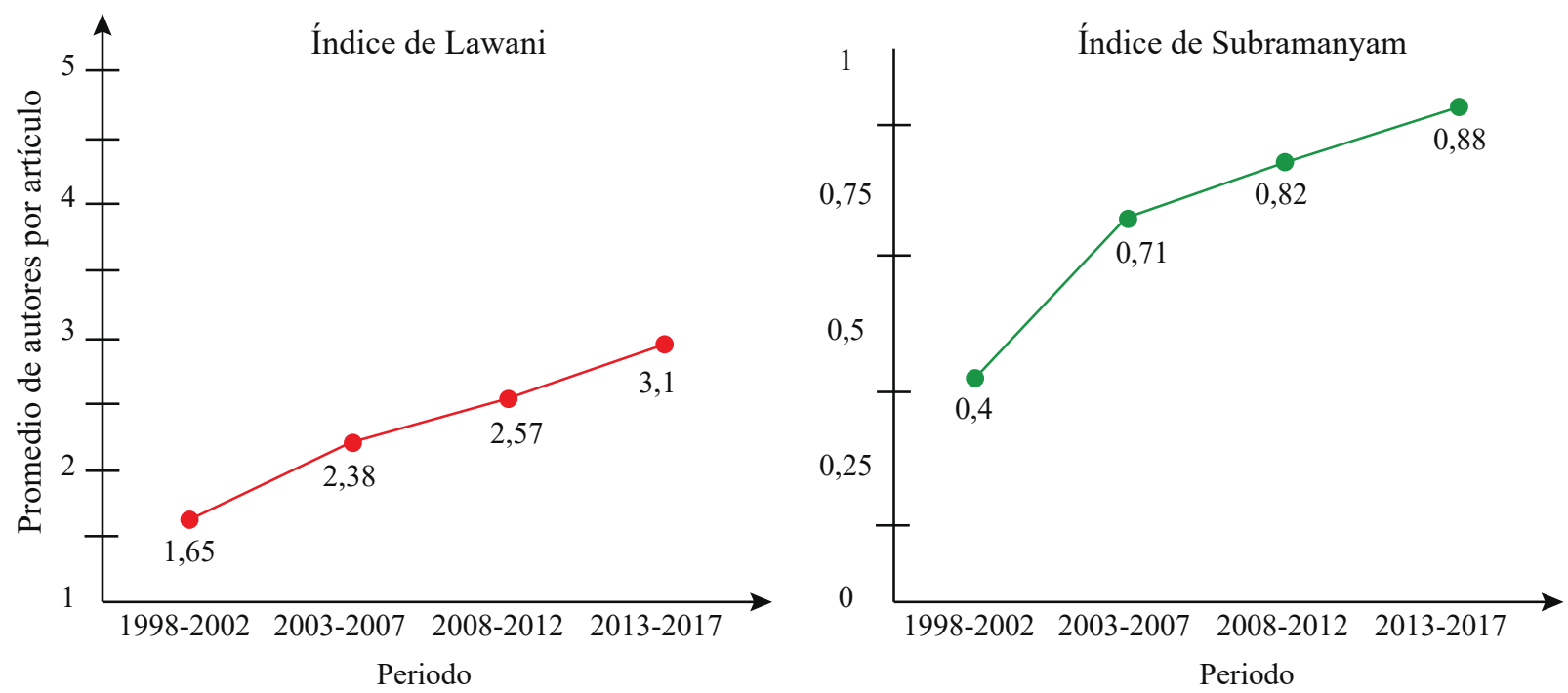

Figura 2. Índice de Lawani e Índice de Subramanyam. Por una parte, el índice de Lawani indica el promedio de autores que firman un artículo -su valor mínimo es 1 y no existe un valor máximo para este índice-; y, por otra, el índice de Subramanyam indica la proporción de artículos con autoría múltiple, en donde se puede obtener un valor entre 0 y 1 -los valores próximos a 1 señalan que los artículos publicados tienen al menos dos autores-.

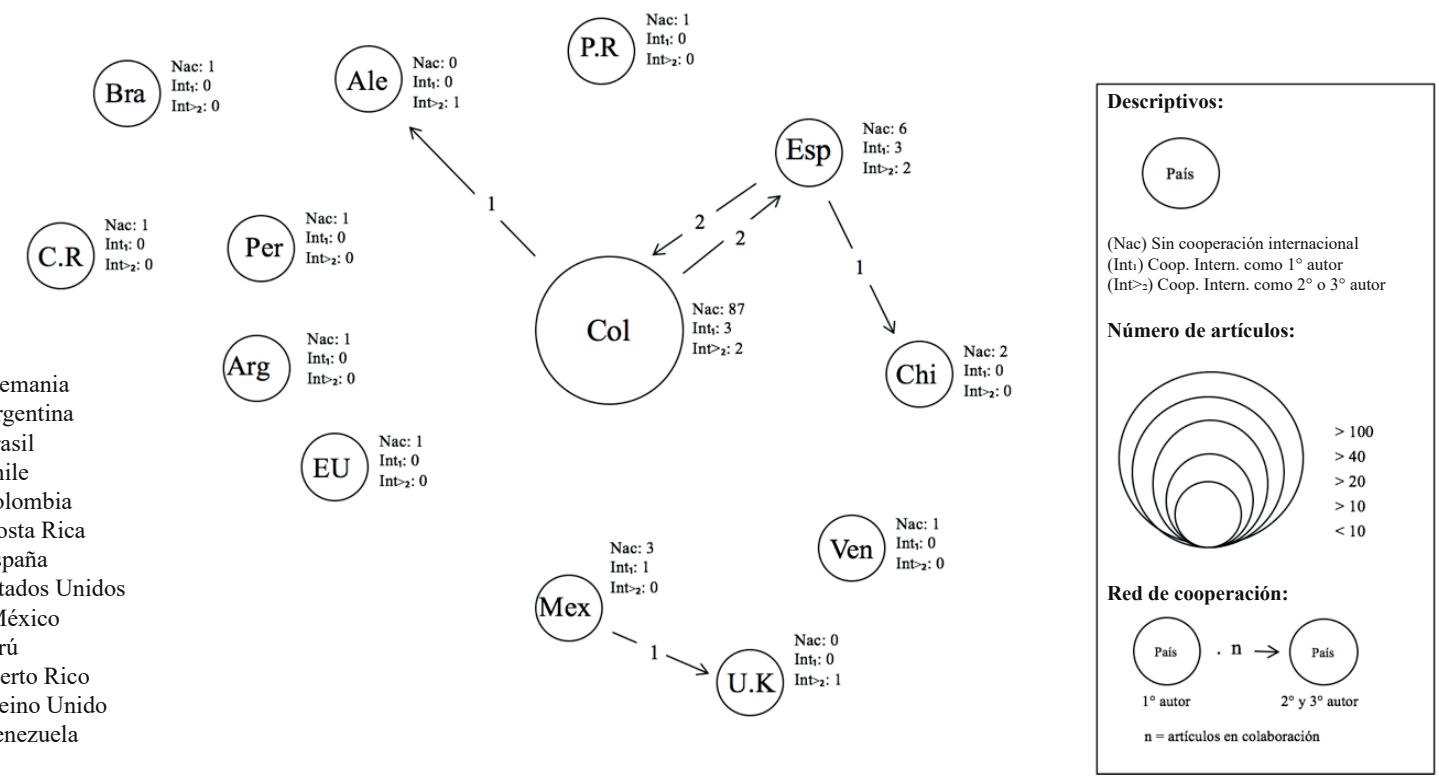

Figura 3a. Mapa de colaboración entre países a partir de los artículos publicados de 1998 a 2007.

entre México y Reino Unido. Como se puede visualizar, las relaciones eran escasas y por lo tanto muy fáciles de identificar, pero, en cambio, en la segunda década el cambio es radical, ya que, si bien en términos de colaboración se mantienen los trabajos realizados entre Colombia y España en primer lugar, la gran cantidad de artículos con al menos alguna afiliación procedente de México ocasiona que este país sea el segundo que desarrolla una importante colaboración con Colombia; aunque también las redes de México se extienden a Cuba, Estados Unidos, España y
Brasil. Asimismo, los aportes de México de igual forma despliegan una importante colaboración interna, aunque esta no fue estudiada en detalle debido a la gran cantidad de instituciones participantes.

\section{Temáticas y metodologías de la investigación}

Las temáticas y metodologías de investigación se encuentran analizadas en términos de la frecuencia y porcentaje en cada uno en los cuatro quinquenios incluidos. En términos de resultados generales, tal como se declaró 


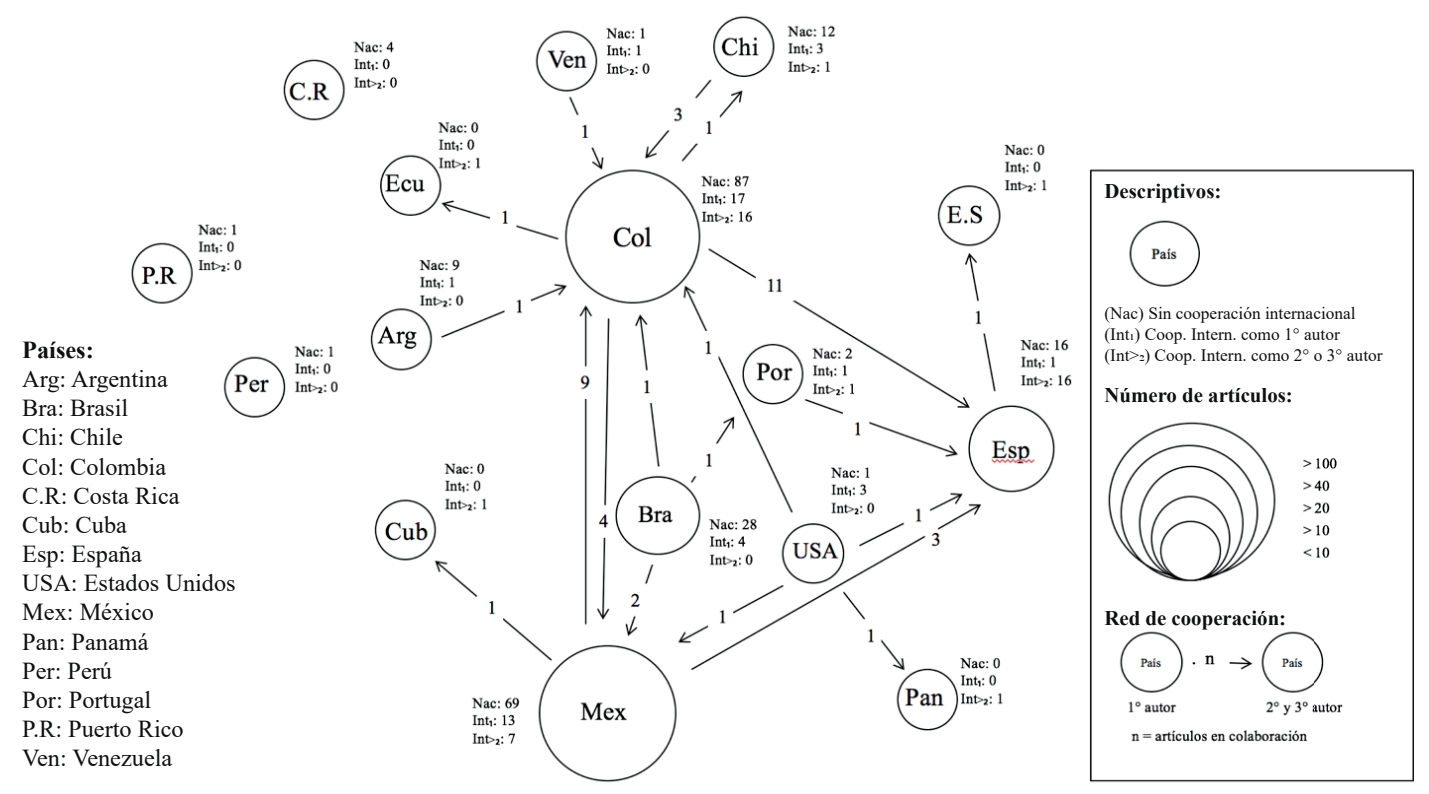

Figura 3b. Mapa de colaboración entre países a partir de los artículos publicados de 2008 a 2017.

en el estudio previo de Ravelo et al. (2016), el área de psicología clínica y de la salud presenta la mayor cantidad de trabajos, seguida por la de psicología educacional, la de psicometría y la de psicología social-comunitaria. Por su parte, la misma situación ocurre con la predominancia de trabajos realizados con metodología cuantitativa (Ravelo et al., 2016), la cual supera ampliamente a los trabajos teóricos y de metodologías cualitativas -los trabajos que incluyen metodologías mixtas son escasos en la trayectoria de la revista-. Adicionalmente, como se puede apreciar (véase Tabla 4), es importante mencionar que, en el último quinquenio, justamente los trabajos en psicología clínica y de la salud y las metodologías cuantitativas también superaron el valor general, lo que demuestra que dichas tendencias no solo son históricas, sino también actuales.

Tabla 4

Temáticas y metodologías de investigación por periodo (quinquenio)

\begin{tabular}{|c|c|c|c|c|c|}
\hline \multirow{2}{*}{ Criterio } & \multicolumn{4}{|c|}{ Periodo } & \multirow{2}{*}{ Total } \\
\hline & $1998-2002$ & $2003-2007$ & $2008-2012$ & 2013-2017 & \\
\hline \multicolumn{6}{|l|}{ Área temática } \\
\hline Ps. Educacional & $15(29 \%)$ & $11(13 \%)$ & $25(20 \%)$ & $20(15 \%)$ & $71(18 \%)$ \\
\hline Ps. Clínica y de la Salud & $14(27 \%)$ & $25(29 \%)$ & $34(28 \%)$ & $42(33 \%)$ & $115(30 \%)$ \\
\hline Ps. Experimental & $8(15 \%)$ & $4(5 \%)$ & $4(3 \%)$ & $6(5 \%)$ & $22(6 \%)$ \\
\hline Neuropsicología & $2(4 \%)$ & $8(9 \%)$ & $10(8 \%)$ & $11(9 \%)$ & $31(8 \%)$ \\
\hline Psicometría & $7(13 \%)$ & $8(9 \%)$ & $14(12 \%)$ & $25(19 \%)$ & $54(14 \%)$ \\
\hline Ps. Jurídica & $6(12 \%)$ & $0(0 \%)$ & $2(2 \%)$ & $1(1 \%)$ & $9(2 \%)$ \\
\hline Ps. Laboral & $0(0 \%)$ & $10(12 \%)$ & $11(9 \%)$ & $6(5 \%)$ & $27(7 \%)$ \\
\hline Ps. Social-Comunitaria & $0(0 \%)$ & $15(17 \%)$ & $17(14 \%)$ & $9(7 \%)$ & $41(10 \%)$ \\
\hline Bibliometría & $0(0 \%)$ & $3(4 \%)$ & $1(1 \%)$ & $3(2 \%)$ & $7(2 \%)$ \\
\hline Otras áreas & $0(0 \%)$ & $2(2 \%)$ & $4(3 \%)$ & $5(4 \%)$ & $11(3 \%)$ \\
\hline \multicolumn{6}{|l|}{ Metodología } \\
\hline Cuantitativa & $30(58 \%)$ & $48(56 \%)$ & $88(72 \%)$ & $109(85 \%)$ & $275(71 \%)$ \\
\hline Cualitativa & $3(6 \%)$ & $16(19 \%)$ & $12(10 \%)$ & $9(7 \%)$ & $40(10 \%)$ \\
\hline Teórica & $18(34 \%)$ & $19(22 \%)$ & $20(16 \%)$ & $3(2 \%)$ & $60(16 \%)$ \\
\hline Mixta & $1(2 \%)$ & $3(3 \%)$ & $2(2 \%)$ & $7(6 \%)$ & $13(3 \%)$ \\
\hline Total & 52 & 86 & 122 & 128 & 388 \\
\hline
\end{tabular}




\section{DISCUSIÓN}

Como se demostró a lo largo del presente artículo, los aportes de Acta Colombiana de Psicología son innegables y muy destacados en la actualidad de la ciencia psicológica de Hispanoamérica. Actualmente, la revista ha subido a la categoría Q3 en Scopus y tiene el desafío de seguir avanzando en la misma ruta. Acá, es importante señalar que los diversos procesos editoriales, así como el apoyo de las autoridades de la revista y de la universidad, han propiciado dichos cambios con efectividad.

Como se ha mencionado, desde el año 2016 todos los artículos están escritos de forma íntegra en inglés (cuando la versión original es español o portugués), también, se ha evidenciado una rápida y eficiente gestión en el proceso de evaluación de pares -según los parámetros de la American Psychological Association- y, finalmente, la política de open access ha permitido una mayor difusión de la revista en diversos sectores de la comunidad científica.

Cabe destacar que para futuros estudios se sugiere analizar los diversos indicadores de citación -algunos datos pueden ser visualizados en el portal SCImago Journal and Country Rank (SCImago, 2018)-; por lo que entre las metas de la revista está mantener e incrementar la internacionalización, aspecto que sin duda será optimizado en el próximo quinquenio. Asimismo, la indexación a la Web of Science es y será la meta natural en este tiempo de consolidación de Acta; y, de lograr esta indexación, se podrá acceder a la publicación de artículos provenientes de otros rincones del mundo, como de países europeos (no hispanoparlantes) y de Oceanía, Asía y África, al mismo tiempo que se intensificará la publicación de trabajos provenientes de América del Norte, lo que genera la apertura de nuevos campos y escenarios de producción.

Por otra parte, no queda ninguna duda de que los avances en la colaboración nacional o internacional potenciarán la citación de la revista. Los resultados evidencian que estas dos variables están estrechamente relacionadas, es decir, que una creciente actividad de colaboración se asocia con un mayor impacto en las citas (Bornmann, 2017). Al respecto, al relevar desde Scopus -con fecha de 26 de marzo de 2018- los diez artículos más citados de la revista (véase Anexo) se puede vislumbrar que ocho de ellos son trabajos desarrollados en colaboración por dos o más autores, y el trabajo más citado (16 citas) es elaborado por un equipo compuesto por cuatro autores (Contreras, Esguerra, Espinoza \& Gómez, 2007).

Finalmente, es importante destacar que el trabajo editorial no culmina en ningún caso con la publicación de los artículos científicos, sino que debe también considerar un nuevo inicio del proceso de investigación (Polanco-Carrasco, 2015) o, más bien, de una etapa que requiere fuertemente de la difusión de la ciencia publicada y sus contenidos. Hoy en día, además de las clásicas y más reconocidas bases de datos de la ciencia (Scopus y Web of Science), es importante destacar plataformas como Academia.edu, Research Gate, Google Scholar, Orcid, Mendeley, y otras que generan mayor visibilidad de los autores y de las revistas en la ciencia actual (Orduña-Malea, Martín-Martín \& Delgado-López-Cozar, 2016). Por esto, se convierte en tarea del Comité Editorial de Acta promover que los investigadores suban sus artículos a dichas plataformas $\mathrm{y}$, por tanto, continuar desarrollando políticas acordes al tiempo que vivimos, tal como se ha hecho desde los inicios de esta prestigiosa revista.

\section{REFERENCIAS}

Ardila, R. (1967). La psicología en Colombia. Interamerican Journal of Psychology, 1(4), 239-249.

Ardila, R. (1998). La Revista Latinoamericana de Psicología: los primeros 30 años. Revista Latinoamericana de Psicología, 30(3), 393-400.

Ardila, R. (2013). Historia de la psicología en Colombia. Bogotá: Manual Moderno.

Ardila, R., \& Pérez-Acosta, A. M. (2010). La psicología colombiana: revistas y bibliografía. Revista Latinoamericana de Psicología, 42(3), 517-523.

Best, D. L., \& Everett, B. S. (2010). The most recent years: The Journal of Cross-Cultural Psychology, 2004-2009. Journal of Cross-Cultural Psychology, 41(3), 329-335.

Bordons, M., \& Zulueta, M. (1999). Evaluación de la actividad científica a través de indicadores bibliométricos. Revista Española de Cardiología, 52(10), 790-800.

Bornmann, L. (2017). Is collaboration among scientists related to the citation impact of papers because their quality increases with collaboration? An analysis based on data from F1000Prime and normalized scores. Journal of the Association for Information Science and Technology, 68(4), 10361047. Doi: 10.1002/asi.23728

Brouwers, S. A., Van Hemert, D. A., Breugelmans, S. M., Van de, \& Vijver, F. J. R. (2004). A historical analysis of empirical studies published in the Journal of Cross-Cultural Psychology 1970-2004. Journal of Cross-Cultural Psychology, 35(3), 251-262.

Buela-Casal, G., \& López, W. (2005). Evaluación de las Revistas Científicas Iberoamericanas de Psicología. Revista Latinoamericana de Psicología, 37(1), 211-217.

Camps, D. (2008). Limitaciones de los indicadores bibliométricos en la evaluación de la actividad científica biomédica. Colombia Médica, 39(1), 74-79. 
Di Bitteti, M. S., \& Ferreras, J. A. (2017). Publish (in English) or perish: The effect on citation rate of using languages other than English in scientific publications. Ambio, 46(1), 121-127. Doi: 10.1007/s13280-016-0820-7

Dominguez Blanco, M. E., \& Duque, C. (1993). La Revista de Psicologia (1956-1991). Revista Colombiana de Psicología, 8, 131-138.

Flórez Alarcón, L. (1998). Centro de Estudios e Investigaciones sobre Adicciones. Acta Colombiana de Psicología, 1, 124-128.

Gallegos, M. (2010). La Revista Latinoamericana de Psicología en sus 40 años de historia. Universitas Psychologica, 9(3), 905-918.

Giraldo, B., \& Rodriguez, O. R. (1997). Historia de la psicología en Colombia: Recuento de algunos de los eventos significativos en los primeros 50 años de historia profesional. Revista de Historia de La Psicología, 18(3/4), 467-485.

Gorbea Portal, S. (2016). Una nueva perspectiva teórica de la bibliometría basada en su dimensión histórica y sus referentes temporales. Investigación Bibliotecológica: Archivonomía, Bibliotecología e Información, 70, 11-16. Doi: 10.1016/j.ibbai.2016.10.001

Gutiérrez, G., Pérez-Acosta, A. M., \& Plata-Caviedes, T. (2009). Desarrollo histórico de una publicación científica: cuarenta años de la Revista Latinoamericana de Psicología. Revista Latinoamericana de Psicología, 41(3), 413-428.

Ho, Y. S. (2014). Classic articles on social work field in Social Science Citation Index: A bibliometric analysis. Scientometrics, 98(1), 137-155.

Lawani, S. M. (1981). Bibliometrics: Its theoretical foundations, methods and applications. International Journal of Libraries and Information Studies, 31, 294-315. Doi: 10.1515/libr.1981.31.1.294

Lonner, W. J. (1980). A decade of cross-cultural psychology: JCCP, 1970-1979. Journal of Cross-Cultural Psychology, $11(1), 7-34$.

López-López, W., \& Calvache, O. (1998). La psicología de habla hispana: 30 años de la Revista Latinoamericana de Psicología. Revista Latinoamericana de Psicología, 30 (3), 401-427.

López-Navarro, I., Moreno, A. I., Quintanilla, M. A., \& ReyRocha, J. (2015). Why do i publish research articles in English instead of my own language? Differences in Spanish researchers'motivations across scientific domains. Scientometrics, 103(3), 939-976. Doi: 10.1007/s11192-015-1570-1

Lotka, J. (1926). The frequency distribution of scientific productivity. Journal of the Washington Academy of Sciences, 16(12), 317-323. Doi: 10.1002/asi.4630280610

Merigó, J. M., Pedrycz, W., Weber, R., \& de la Sotta, C. (2018). Fifty years of Information Sciences: A bibliometric overview. Information Sciences, 432, 245-268. Doi: 10.1016/j. ins2017.11.054
Milfont, T. L., \& Page, E. (2013). A bibliometric review of the first thirty years of the Journal of Environmental Psychology. Psychology, 4(2), 195-216.

Montero, I., \& León, O. G. (2007). A guide for naming research studies in psychology. International Journal of Clinical and Health Psychology, 7, 847-862.

Morales Quintero, L. A. (2008). Reseña bibliográfica: Neurociencia, Violencia y Sistema de Justicia. Acta Colombiana de Psicología, 11(1), 167-168.

Orduña-Malea, E., Martín-Martín, A., \& Delgado-LópezCozar, E. (2016). La bibliometría que viene: Almetrics (Author-Level Metrics) y las múltiples caras del impacto de un autor. El Profesional de la Información, 25(3), 485-496.

Oviedo, G. L. (2012). Colombian approaches to psychology in the 19th century. History of Psychology, 15(4), 291-301.

Pérez-Acosta, A. M., \& Perilla Toro, L. E. (1996). Colombian Psychology through its scientific journals. International Journal of Psychology, 31(3-4), 159.

Pérez-Acosta, A. M., \& Perilla Toro, L. E. (2006). Una retrospectiva de la psicología en Colombia. Bogotá: Psicom Editores.

Pire, R. (2015). La importancia de las revistas científicas universitarias y la necesidad de su financiamiento. Compendium, 18(35), 1-2.

Pritchard, A. (1969). Statistical bibliography or bibliometrics? Journal of Documentation, 25, 348-349.

Polanco-Carrasco, R. (2015). Editar para perturbar. Cuadernos de Neuropsicología, 9(3), 10-11. Doi: 10.7714/cnps/9.3.101

Polanco-Carrasco, R., Gallegos, M., Salas, G., \& López-López, W. (2017). Las revistas de psicología en Chile: historia y situación actual. Terapia Psicológica, 35(1), 81-93.

Ravelo, E., Mejía, S., \& González, M. (2016). La producción científica de Acta Colombiana de Psicología: Análisis descriptivo del período 2010-2014. Acta Colombiana de Psicología, 19(2), 281-297.

Ravelo, E. (2017). Editorial Revista Acta Colombiana de Psicología. Acta Colombiana de Psicología, 20(1), 7.

Salas, G. (2014). El Informe Nassar (1955) sobre la formacion de psicólogos en Chile. Revista de Psicología, 13(1), 109112. Doi: 10.5354/0719-0581-2014.32879

Salas, G., Ponce, F. P., Méndez-Bustos, P., Vega-Arce, M., Pérez, M. A., López-López, W., \& Cárcamo-Vásquez, H. (2017). 25 años de Psykhe: Un análisis bibliométrico. Psykhe, 26(1), 1-17. Doi: 10.7764/psykhe.26.1.1205

SCImago Journal and Country Rank. (2018). Acta Colombiana de Psicología. Recuperado de http://www.scimagojr.com/ journalsearch.php? $\mathrm{q}=10600153356 \&$ tip $=$ sid \& clean $=0$

Sebastiani, R. W., \& Maia, E. M. C. (2006). Análise da evolução da produção científica apresentada em eventos científicos de psicologia da saúde na América Latina (1985-2003). Acta Colombiana de Psicología, 9(1), 5-24. 
Spinak, E. (1998). Indicadores cienciométricos. Acimed, 9(2), 42-49.

Subramanyam, K. (1983). Bibliometric studies of research collaboration: A review. Journal of Information Science, 6, 3338. Doi: $10.1177 / 016555158300600105$

Sweileh, W. M. (2017). Bibliometric analysis of medicinerelated publications on refugees, asylum-seekers, and internally displaced people: 2000-2015. BMC International Health and Human Rights, 17(1), art. n. ${ }^{\circ} 7$.

Sweileh, W. M., Al-Jabi, S. W., Sawalha, A. F., AbuTaha, A. S., $\&$ Zyoud, S. H. (2016). Bibliometric analysis of medicinerelated publications on poverty (2005-2015). SpringerPlus, 5(1), art. n. ${ }^{\circ} 1888$.

Tsay, M. Y., \& Li, C. N. (2017). Bibliometric analysis of the journal literature on women's studies. Scientometrics, 1-30. Artículo en prensa. Doi: 10.1007/s11192-017-2493-9
Urbizagástegui, R. (2005). La productividad científica de los autores. Un modelo de aplicación de la Ley de Lotka por el método del poder inverso generalizado. Información, Cultura y Sociedad, 12, 51-73.

Vargas, C. (1998). Editorial: Revista Acta Colombiana de Psicología. Acta Colombiana de Psicología, 1, 5-6.

Vargas, C. (2004). Información institucional: Reconocimiento de Colciencias para Acta Colombiana de Psicología. Acta Colombiana de Psicología, 9, 121.

Vera-Villarroel, P., López-López, W., Lillo, S., \& Silva, L. M. (2011). La producción científica en psicología latinoamericana: Un análisis de la investigación por países. Revista Latinoamericana de Psicología, 43(1), 95-104.

Zych, I., \& Buela-Casal, G. (2010). Internacionalidad de las revistas de psicología multidisciplinar editadas en Iberoamérica e incluidas en la Web of Science. Universitas Psychologica, 9(1), 27-34. 


\section{ANEXO}

Artículos más citados en Acta Colombiana de Psicología (1998-2017)

Título
Validación colombiana del sistema internacional de imágenes afectivas: Evidencias del origen transcultural de la emoción.

Validación del Body Shape Questionnaire (Cuestionario de la Figura Corpo- Castrillón Moreno, Luna Montaño, ral) BSQ para la población Colombiana.

Creencias y conductas ambientales, liberalismo económico y felicidad.

Optimismo y salud positiva como predictores de la adaptación a la vida universitaria.

Asertividad, resistencia a la presión de grupo y consumo de alcohol en universitarios.

Panorama general de la producción académica en la psicología colombiana indexada en psicoredalyc, 2005-2007.

Burnout: Síndrome de quemarse en el trabajo (SQT)

Desempeño académico de universitarios en relación con ansiedad escolar y autoevaluación.

Habilidades prosociales, rasgos de personalidad de género y aceptación de la violencia hacia la mujer en adolescentes que han presenciado violencia entre sus padres.
Autor(es) y año de publicación

Contreras, Esguerra, Espinosa \&

Gómez (2007).

Gantivia, Guerra \& Vila (2011).

Número de citas Avendaño Prieto \& Pérez-Acosta (2007).

Moyano-Díaz, Cornejo \& Gallardo (2011).

Londoño (2009).

Londoño Pérez \& Valencia Lara (2008).

López, Silva, García-Cepero, AguilarBustamante \& Aguado (2010).

Quiceno \& Vinaccia (2007).

Hernández-Pozo, Coronado Alvarez, Araújo Contreras \& Cerezo Reséndiz (2008).

Rey (2008). 